\title{
PROPUESTA METODOLÓGICA BASADA EN COMPETENCIA PARA LA ENSEÑANZA DE GRÁFICAS Y ANÁLISIS DE FUNCIONES TRIGONOMÉTRICAS
}

\author{
Blanca R. Gómez R. ${ }^{[1]}$, \\ Moisés Medina $\mathrm{L}^{[2]}$., \\ Eugenio C. López M. ${ }^{[3]}$
}

\section{Resumen}

El objetivo de esta investigación es elaborar una propuesta metodológica para la enseñanza de las funciones trigonométricas mediante el enfoque de competencias, en el cuarto año (décimo grado) de Educación Secundaria, en ella se analiza el nivel que poseen los estudiantes en este tema y se anexa una unidad didáctica para impartir este contenido.

Para la realización de este trabajo de carácter explorativo, se usaron los enfoques cualitativos y cuantitativos. Las técnicas utilizadas fueron la encuesta, la entrevista a informantes claves y la observación.

Entre los resultados más relevantes de esta investigación está en que todos los estudiantes a los cuales se les aplicó el examen experimental no pueden graficar funciones trigonométricas; además, se les hace muy difícil analizar la solución de ejercicios por lo que no recuerdan las definiciones de las funciones trigonométricas.

Se concluye que se hace necesaria de la utilización de nuevas metodologías de enseñanza para impartir este tema por lo que anexamos una unidad didáctica para la enseñanza de este tema.

\section{Introducción}

Esta investigación titulada: Propuesta metodológica basada en competencia para la enseñanza de las gráficas y el análisis de las funciones trigonométricas. En el cuarto año diurno del colegio "Francisco Aarón Joce Flores" de la Colonia "Naciones Unidas", primer semestre del año 2009; en la ciudad Nueva Guinea, RAAS, Nicaragua. Está diseñado específicamente para mejorar la enseñanza aprendizaje, así también servirá como una herramienta didáctica para los docentes que vayan a desarrollar dichos contenidos; ya que podrá utilizar los procesos didácticos que aquí se detallan.

[1] Licda. Matemática. URACCAN, Nueva Guinea. Reyesblanca7@yahoo.es

[2] Lic. Matemática. URACCAN, Nueva Guinea. Sesmoizoo7@yahoo.com

[3] Msc. Matemática. URACCAN, Nueva Guinea. Elmsmp20o2@yahoo.es 
Los estudiantes de cuarto año, del colegio "Francisco Aarón Joce Flores" de la Colonia Naciones Unidas, presentan serias dificultades para comprender, resolver y analizar las gráficas de las funciones trigonométricas, así como las características particulares de cada una y sus propiedades, porque los docentes que las imparten no hacen uso de las herramientas necesarias para su desarrollo, tales como medios didácticos que mejoren el aprendizaje y actividades necesarias que sirvan para crear un aprendizaje significativo.

Otro motivo por lo cual los estudiantes no aprenden los temas en cuestión es por el poco interés que tienen para hacerlo, tomándolo como una actividad impuesta como requisito para aprobar la asignatura.

Es importante señalar que actualmente en el gremio educativo ya se está trabajando con el enfoque por competencia. Éste enfoque tiene como propósito que los estudiantes sean capaces de desarrollar competencias básicas que les permitan alcanzar el nivel deseado por las universidades. Pero por falta de capacitación y modelos de enseñanzas para los docentes de educación tanto primaria como secundaria, no se ha estado trabajando con la intensidad y plenitud que tal enfoque posee.

Para la realización de este trabajo fue necesario analizar la problemática que presentan los estudiantes para el aprendizaje de las funciones trigonométricas. También se caracterizó el nivel actual de conocimiento que tienen estos estudiantes en cuanto al tema en cuestión, y para poder dar respuesta a la problemática encontrada se brinda una propuesta de aprendizaje basada en competencias para la enseñanza.

Para tal fin se analizaron tesis elaborados por matemáticos graduados en la URACCAN. Éstas propuestas estaban basadas en el enfoque constructivista, las cuales tienen como fin contribuir al mejoramiento de la enseñanza aprendizaje.

Para la realización de esta investigación se hizo uso del enfoque mixto, debido a que se analizan datos cualitativos y cuantitativos, pero hay dominio del enfoque cualitativo debido a las características del trabajo. 


\section{Revision de Literatura}

\section{Conceptos Fundamentales en la enseñanza de las matemáticas}

"La enseñanza es una actividad realizada conjuntamente mediante la interacción de tres elementos: un profesor o docente, uno o varios estudiantes o discentes y el objeto de conocimiento". (Wikipedia, 2009). De acuerdo con las concepciones más actuales, cognitivistas, el docente actúa como "facilitador", "guía" y nexo entre el conocimiento y los estudiantes, logrando un proceso de interacción, (antes llamado proceso "enseñanza-aprendizaje"), basado en la iniciativa y el afán de saber de los discente; haciendo del proceso una constante, un ciclo e individualizando de algún modo la educación.

Aprendizaje: es aquel que va en pro de los fortalecimientos de todas aquellas actitudes biopsicosocioafectivas de los seres humanos a través de la aplicación de estrategias basadas en la apreciación de la realidad por medio de las experiencias propias y lógicas y los canales sensoriales. (Rodríguez, 2003).

Matemática: es el nombre que le damos a la colección de todas las pautas e interrelaciones posibles. Algunas de estas pautas son entre formas, otras en secuencia de números, en tanto que otras son relaciones más abstractas entre estructuras. La esencia de las matemáticas está en la relación entre cantidades y cualidades. (Barrow J.D , 2008)

\section{La enseñanza de las matemáticas y sus problemáticas}

"Aprendemos cuando comprendemos, explicamos, aplicamos, criticamos. En todos estos procesos subyace la memoria que nos permite recordar el conocimiento". Quesada (2007 p.15).

\section{Los cambios curriculares}

Formar a todos los niños, las niñas, adolescentes, jóvenes y adultos, sujetos de la Educación Básica y Media, para el desempeño exitoso de su vida personal, social, cultural, ambiental y laboral que contribuya al desarrollo humano sostenible; así como para la continuación eficaz de sus estudios formales y no formales. (MINED, 2007, p. 45)

"Abarca el desarrollo de las actitudes de la persona, lo que el individuo es en su afectividad y su voluntad, buscando un enfoque integrador en que la persona, desde su ser, ponga en juego todo su saber y su saber hacer".(Gil R.J, 2005). 


\section{CIENCIAS SOCIALES}

\section{Enfoque por competencia}

En nuestro trabajo investigativo se trabajó en el enfoque por competencias entiéndase como "competencia a la a capacidad de afrontar un problema complejo, o de resolver una actividad compleja". (Godino J.D \& Rivas M. et al,2008, p.1).

Competencia es: "La capacidad para entender, interpretar y transformar aspectos importantes de la realidad personal, social, natural o simbólica". Cada competencia es así entendida como la integración de tres tipos de saberes: "conceptual (saber), procedimental (saber hacer) y actitudinal (ser)". (Gil R.J, 2005).

Cepeda, J. M (1681), clasifica el proceso de la metodología de la enseñanza en seis momentos o tipos de evaluación diseñada con el enfoque por competencia: "Evaluación de necesidades especificas de competencias, determinación de componentes y niveles de realización, identificación de procedimientos para el desarrollo de competencias, definición de evaluación de competencias, y validación de competencias."

\section{El estudiante}

Quesada (2007, p, 96) "el buen estudiante no nace, se hace, he aquí el secreto. El verdadero aprendizaje, el que perdura, se logra mediante el proceso que transforma el contenido de la enseñanza de manera que adquiere sentido y significado para el estudiante"

\section{Problemática del aprendizaje.}

Mucha gente se siente boba, o torpe, o sin capacidades para las matemáticas su pasado está lleno de todo tipo de problemas con las matemáticas, que se traducen en humillaciones, decepciones, profesores regañones y temibles exámenes. A mujeres, en algunas ocasiones se les ha dicho de manera mordaz que es normal que no sean buenas para las matemáticas (Seiter, 1996, p. 187).

La capacidad para comprender los temas que se estudian no se debe al género, raza, religión, cultura, a la que pertenezca un estudiante sino, que se debe esencialmente a dos causas principales: la primera por el "poco esfuerzo" intelectivo que se le da, por falta de interés cuando se estudia, segundo por la "debilidad" por la capacidad de la mente que asume trabajo que le exceden a sus capacidades. (Bosco, 2003, p, 115). 


\section{Análisis de las funciones trigonométricas y sus graficas}

Para comprender estas funciones trigonométricas es de vital importancia memorizar y entender las principales características de las funciones transcendentales, las que según Wikipedia (2008).

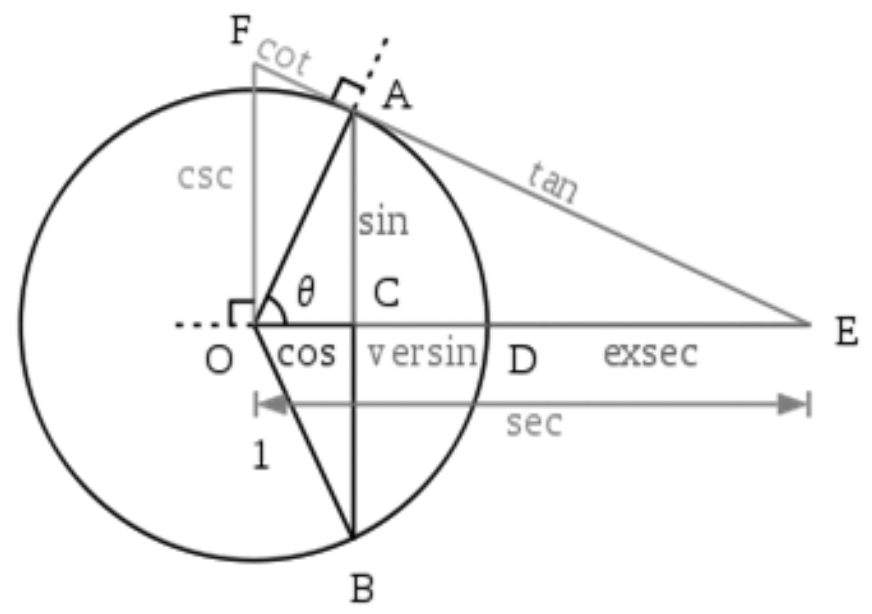

"Todas las funciones trigonométricas de un ángulo $\theta$ pueden ser construidas geométricamente en términos de un círculo unidad centrado en $\mathrm{O}$ ".

También mostramos el siguiente esquema. Donde se observa las relaciones que se pueden establecer entre las funciones trigonométricas (Wikipedia, 2008).

\section{Materiales y métodos}

Fue realizada con los estudiantes del cuarto año del colegio "Francisco Aarón Joce Flores" de la "Colonia Naciones Unidas", Nueva Guinea. Fue realizada en los meses de junio a agosto, 2009. El enfoque utilizado es el mixto, es de carácter explorativa, de corte trasversal y la muestra utilizada es de 13 estudiantes debido a que el cuarto año de este colegio sólo tenían 13 estudiantes, por tanto la muestra tiene el 100 por ciento de la población.

\section{Métodos y técnicas utilizadas}

Las fuentes primarias de esta investigación fueron: las entrevistas dirigidas a estudiantes y docente, el grupo focal, la encuesta y el test aplicada a los estudiantes del cuarto año, así como observaciones directas a pie de aulas durante el proceso enseñanza, con el fin de valorar las estrategias utilizadas por el profesor y luego brindar sugerencias y estrategias diversas que faciliten la enseñanza del tema de las funciones trigonométricas. 


\section{Las variables}

Las principales variables de este estudio fueron: Metodología y Materiales didácticos utilizados por el docente para impartir la clase de matemáticas y principalmente nuestro tema en estudio, distractores, Interés mostrado a la clase de matemáticas, horas que dedica para hacer la tarea, nivel de comprensión del tema.

\section{Resultados y discusión}

Este estudio se dividió en cuatro partes fundamentales tratando de detallar cada aspecto y ordenar la información de manera comprensible y clara.

\section{Caracterización de la enseñanza en el colegio "Francisco Aarón Joce Flores"}

En este estudio se encontró que el docente que imparte la asignatura de matemáticas es muy dinámico, lo que permite que los estudiantes estén motivados y por consiguiente se interesen por aprender; sin embargo, según la observación se logró constatar que hace falta mas explicación para que los estudiantes se apropien de los algoritmos a seguir para desarrollar los contenidos, además debe utilizarse metodologías de enseñanza tales como: estudiantes monitores, trabajos en equipos, atención individual, entre otras.

\section{Caracterización del aprendizaje}

La edad de los estudiantes interfiere en el aprendizaje, debido a que existe poco interés por aprender los contenidos matemáticos, además que no tienen el hábito de realizar las tareas, lo cual perjudica de manera directa en el aprendizaje, ya que no se retroalimenta la clase de manera individual, a saber que la manera eficaz de aprender es la práctica.

\section{Caracterización de la comprensión del tema de las gráficas y las funciones trigonométricas por parte de los estudiantes}

Aunque los estudiantes se consideran buenos en matemáticas, los resultados obtenidos por el examen experimental fueron muy deficientes; ya que no fueron capaces de resolver ni la mitad de los ejercicios propuestos; lo que demuestra que estos estudiantes tienen muchas dificultades para resolver ejercicios de trigonometría y entender las principales características de las funciones trigonométricas. 


\section{Niveles de conocimientos que presentan los estudiantes en el contenido de las gráficas y el análisis de las funciones trigonométricas}

Los estudiantes no se saben las definiciones de razones trigonométricas, ni son capaces de hacer uso de ellas en la resolución de ejercicios que impliquen las aplicaciones con este tipo de razones, además de no ser capaces de resolver ejercicios aplicando estos conceptos, igualmente no sabe identificar las gráficas de las funciones trigonométricas, por consiguiente no identifican los nombres de las razones trigonométrica teniendo la gráfica.

\section{Conclusiones}

Luego de analizar las encuestas y entrevista, se a llegado a las siguientes conclusiones.

- El profesor de la escuela “Francisco Aarón Joce Flores” es dinámico, opinión confirmada por el 92 por ciento de los estudiantes encuestados. Por tanto motiva a los estudiantes, y utiliza algunos métodos que le permiten conquistar el interés de los estudiantes en los temas que desarrollan.

- $\quad$ El 69 por ciento de los estudiantes contestaron que el maestro de matemática no hace apoya en estudiantes monitores, lo que no permite atender a todos los estudiantes.

- El 92 por ciento de los examinados opinan que el maestro asigna trabajos en equipos lo que permite una mejor socialización de las actividades, pero por la falta de atención individual les dificulta la realización de éstas a los estudiantes.

- Gran parte de los encuestados afirman que su entendimiento en el tema de las funciones trigonométricas es poco, lo cual está representado por el 85 por ciento del total.Este resultado concuerda con los obtenidos en el examen experimental donde ninguno de los estudiantes pudieron graficar las funciones que se les indicó resolver,

- Los estudiantes consideran poco o nada útil el tema investigado para su futuro (un 54\%), por lo que existe poca motivación por parte del maestro.

- El 31 por ciento los estudiantes no comprende los contenidos por falta de atención a las asignaturas, por lo que es necesario la aplicación de clases más dinámicas, que sean capases de captar la atención del educando.

- El 62 por ciento de los examinados realiza con frecuencia la tarea, pero por la falta de quien les ayude muchas veces se equivocan en la solución de ésta.Aunque un 46 por ciento de los estudiantes afirma entenderle al tema de las funciones trigonométricas los resultados del examen experimental niegan esta afirmación, por lo antes expresado. 


\section{CIENCIAS SOCIALES}

- Gran parte de los investigados consideran el tema de las funciones trigonométricas asequible, lo que está representado por el 61 por ciento. Sin embargo los resultados de la prueba realizada nos demuestra la necesidad una nueva forma de enseñar, que involucre a los estudiantes para conseguir un mayor interés por parte de ellos.

- A muchos de los educandos les gusta poco o nada la asignatura de matemática, (64\%), lo que influye en el poco aprendizaje de la misma.

- 77 por ciento de los estudiantes no fueron capases de encontrar las razones trigonométricas partiendo de una de ellas como dato, lo que demuestra el poco dominio de los concepto de las razones trigonométricas.

- 77 por ciento de los analizados fueron capaces de encontrar el resultado de las razones trigonométricas estando expresadas éstas en radianes, pero ninguno de ellos fue capaz de resolver los ejercicios estando el argumento en grados lo que demuestra que no saben la diferencia entre radianes y grados, tampoco el uso correcto de la calculadora.

- Ninguno de los estudiantes conocían las características de las funciones trigonométricas ya que no respondieron la pregunta. Para qué valor de $\mathrm{x}$ se satisface $\sin \mathrm{x}=\sqrt{5}$.

- Ninguno de los estudiantes fue capaz de graficar las funciones trigonométricas, ni de reconocer las presentadas.

\section{Lista de referencias}

Barrow J.D. (2008,) Definiciones de Matemática recuperado 15 de Mayo, en http://www. epsilones.com/paginas/t-definiendo.html

Bosco J. (2003). EDUCERE. Managua, Nicaragua.

Gil R.J. (2005) Enseñanza para la Comprensión, EpC, para una formación en competencias. Recuperada 14 de Mayo en http://impreso.elnuevodiario.com.ni/2006/o1/15/ opinion/10297

Godino J.D \& Rivas M. et al, (2008). Competencias del profesor de matemáticas Recuperado el 15 de Marzo, en http://www.ugr.es/ jgodino/funciones-semioticas/competencias_anadida_24junioo8.pdf

MINED (2007) Proyecto Excelencia. Recuperado el.12 de Agosto, 2009 en http:// www.mined.gob.ni/PDFo7/boletin\%2oEXCELENCIA-1\%2oindd.pdf Contribucionesv3n1002/ funcion exponencial/ index.html.

Wikipedía (2010), la enciclopedia libre, La enseñanza, modificada por última vez el 1 jun 2010, Recuperada 12 Junio 2010. 
Quesada R (2007). Ejercicios para mejorar la memoria. Primera edición. México.

Rodríguez J. Definiciones y tipos de aprendizajes significativos Recuperado 23 de Junio,en http://www.cprceuta.es/Asesorias/FP/Archivos/FP\%2oDidactica/ Definiciones\%20y\%20tipos\%20de\%20aprendizaje\%20significativo.pdf

Ruiz, A (2003). Historia y filosofía de las Matemáticas. EUNED, San José, Costa Rica

Sanabria, G (2006). Las Funciones Trigonométricas con el Método de Exhaución de Arquimedes: Dos propuestas metodológicas. Universidad de Costa Rica (UCS). Tesis.

Sánchez E. (2009). El estudio activo de trabajo intelectual. Recuperado el 18 septiembre, 2009 en http://www.slideshare.net/adrysilvav/ tecnicas-de-estudios-universitarios-a-distancia

Sequeira, C V,\& Cruz, P A. (200o). Manual de investigación: investigar es fácil, UNAN, Managua, pp. 107.

Setter Ch. (1996). Matemática de uso diario para DUMMIES, primera edición.pp. o6 